\title{
Isolation and Gharacterization of Group B Streptococci from Genito-Urinary Tracts in Japan
}

\author{
Nobuhisa Yamane, Masako Yuki and Koichi Kyono* \\ Clinical Microbiology Branch, Central Clinical Laboratory, \\ and * Department of Obstetrics and Gynecology, Tohoku \\ University School of Medicine, Sendai 980
}

\begin{abstract}
Yamane, N., YukI, M. and Kyono, K. Isolation and Characterization of Group B Streptococci from Genito-Urinary Tracts in Japan. Tohoku J. exp. Med., 1983, 141(3), 327-335 — The prevalence of group B streptococci in urine specimens submitted from both inpatients and outpatients to the Clinical Microbiology Laboratory were investigated. Out of 3,780 urine specimens, forty-seven strains were identified as group B streptococci, and clinical features revealed that the isolation ratio from outpatients was higher than that from inpatients, and that the majority of isolates were from female urine. Whereas the isolation ratios of group B streptococci from vaginal swabs were $2.9 \%$ in 377 pregnant women at 37-40 weeks of gestation, and $4.8 \%$ in 335 non-pregnant women. Also, the newborns delivered from mothers tested were investigated. Only two cases were positive in ear, pharyngeal and/or nasal swabs, but no clinical manifestation was observed. An alternative nomenclature system of group B streptococci based on the serological classification of both heat-labile protein and heat-stable polysaccharide antigens was evaluated for the clinical isolates and compared with the original Lancefield's procedure. The results indicated that the method described will provide a simple and reasonable technique for serotyping of group B streptococci. group B streptococci; urine; vaginal swabs; pregnancy; serotype
\end{abstract}

It is well known that group B streptococci (GBS), Streptococcus agalactiae, are the most common cause of neonatal sepsis and/or meningitis, elevating the neonatal mortality and morbidity ratios in spite of current antibiotic therapy (Eickhoff et al. 1964; McCracken and Shinefield 1966; Franciosi et al. 1973; Hall et al. 1976; Gardner et al. 1979), and that GBS are widely prevalent in the birth canal (Butter and de Moor 1967; Anthony et al. 1978, 1981). These authors emphasized the association of GBS in the vaginal flora and perinatal infections. However, it is also reported that the prevalence of GBS is highly erratic, reflecting differences in human genetic constitution, in sexual practice or in environmental factors such as hygiene and nutrition (Anthony et al. 1978; Collado et al. 1981). There were found several reports concerning the prevalence of GBS in pregnant women and neonatal infections in Japan (Murai et al. 1980; Ohashi 1981). However, the isolation ratios of GBS from vaginal swabs in them were low and the occurrence of

Received for publication, March 22, 1983. 
neonatal septicemia and/or meningitis with GBS in Japan were very limited in comparison with those in other countries. This difference observed in Japan and Western countries led us to conduct the epidemiological study of GBS in genitourinary tract using a more sensitive and selective medium for GBS. Here we will describe the prevalence of GBS in cervix swabs of pregnant and non-pregnant women, and urine taken from patients of Tohoku University Hospital, Sendai.

Up to this time, the hot-acid extraction procedure originally established by Lancefield (1934) has been commonly employed for the serotyping of GBS. However, this method is time-consuming and complex. Recently, an alternative procedure for serotyping GBS using the slide agglutination method modified from the method for serotyping group A streptococcus $\mathrm{T}$ antigen is being developed (personal communication from Denka Institute of Biological Sciences, Ltd., Tokyo). This procedure is based on the qualitative assay of both heat-labile (protein) and heat-stable (polysaccharides) antigens of GBS. In our communication, we will describe the results obtained by this alternative method and compared them to Lancefield's hot-acid extraction procedure.

\section{Materials and Methods}

Specimens for GBS isolation. A total of 377 cervical swabs were collected from pregnant women attending the Department of Obstetrics and Gynecology, Tohoku University School of Medicine, from October 1981 to June 1982. The specimens were taken with cotton swabs from women in their 37-40th week of gestation. Besides cervical swabs, throat, ear and nasal swabs were also collected from their newborns within one day after delivery. Also, a total of 335 cervical swabs collected from non-pregnant women for bacterial and/or fungal examination were tested for GBS. Urine specimens from the patients of Tohoku University Hospital from September 1981 to June 1982 were also included in this study.

Laboratory examination. All the swabs taken were directly inoculated into $3 \mathrm{ml}$ of selective broth medium (Todd-Hewitt broth containing $5 \%$ of sheep blood, $15 \mu \mathrm{g} / \mathrm{ml} \mathrm{of}$ nalidixic acid and $8 \mu \mathrm{g} / \mathrm{ml}$ of gentamycin). After overnight incubation at $37^{\circ} \mathrm{C}$, the cultures in broth medium were transferred onto fresh $5 \%$ sheep blood agar and then incubated for another $18 \mathrm{hr}$. The colonies identified as Gram positive cocci and showing beta- or nonhemolysis on sheep blood agar were first tentatively identified by the following physiological tests; hippurate hydrolysis, Christie, Atkins, and Munch-Peterson(CAMP) test, bacitracin susceptibility and bile esculin hydrolysis (Centers for Disease Control 1977a). Then, the strains were serologically classified using the Phadebact Streptococcus Test Kit (Pharmacia Diagnostics, Uppsala, Sweden). The urine specimens were quantitatively examined by automicrobic system MS-2 (Abbott, Dallas, USA) using eugonic broth. After $5 \mathrm{hr}$ incubation at $35^{\circ} \mathrm{C}$, the positive cultures $\left(10^{5}\right.$ colony forming units $/ \mathrm{ml}$ or more $)$ were transferred onto $5 \%$ sheep blood agar, and then characterized as described above.

The strains isolated and identified as GBS were first grouped serologically according to the procedure of Lancefield's hot-acid extraction (1934) and capillary precipitin modified by Centers for Disease Control (1977b) with antisera to types Ia, Ib, Ic, II, III, R and X. The antisera were kindly donated by Dr. J. Rotta, WHO Collaborating Center for Reference and Research on Streptococci, Prague, Chechoslovakia, and Dr. R.R. Facklam, Centers for Disease Control, Atlanta, USA. For the serotyping of heat-labile and heat-stable antigens of GBS, the method recently proposed by the Denka Institute of Biological Sciences, Ltd. (Tokyo) was employed. The isolates were incubated into $5 \mathrm{ml}$ of Todd-Hewitt broth overnight at $30^{\circ} \mathrm{C}$, and the pellet was collected after low speed centrifugation. The pellet was resuspended in $0.5 \mathrm{ml}$ of Todd-Hewitt broth and then digested with pig pancrease extract at $\mathrm{pH}$ 8.0 for $60 \mathrm{~min}$ at $37^{\circ} \mathrm{C}$. After low speed centrifugation, the pellet was resuspended into $0.5 \mathrm{ml}$ 
TABLE 1. Antisera preparations against heat-stable and heat-labile antigens of group $B$ streptococcus

\begin{tabular}{cll}
\hline Antisera to; & \multicolumn{1}{c}{ Reference strain } & Serotype \\
\hline Heat-stable antigen & & \\
Ia & 090R (Lancefield) & Ia \\
Ib & R36B (Lancefield) & Ib/S \\
II & 18RS21 (Lancefield) & II \\
III & 6313 (Jelinková) & III \\
IV & FZ 607 (Fuji Zoki) & IV/Q \\
V & FZ 314 (Fuji Zoki) & V \\
Heat-labile antigen & & \\
S & FZ 301 (Fuji Zoki) & Ia/S \\
X & Compton (Pattison) & X \\
R & 090R variant (Pattison) & R \\
Q & FZ 345 (Fuji Zoki) & Ia/Q \\
W & FZ 450 (Fuji Zoki) & W \\
\hline
\end{tabular}

of phosphate buffer saline (PBS, $\mathrm{pH}$ 7.4). The suspension was then employed as a heat-labile antigen of GBS for the agglutination test against each antiserum. Then the suspension was autoclaved at $120^{\circ} \mathrm{C}$ for $30 \mathrm{~min}$, and tested against each antiserum to the heat-stable antigen of GBS. The antisera employed in the study were donated by Denka Institute of Biological Sciences, Ltd., Tokyo, and are listed in Table I.

Susceptibility to antibiotics was determined by the agar-dilution method standardized by the Japan Society of Chemotherapy (1981) using $10^{6}$ colony forming units per ml of organisms. The minimum inhibitory concentration (MIC) was assayed on horse blood agar.

\section{Results}

\section{GBS isolation from urine}

A total of 3,780 urine specimens were quantitatively examined by MS-2 urine screening tests using eugonic broth. Out of these, 1,287 samples were positive. One thousand nine hundred and twenty-nine strains were isolated from these positive cultures. Forty-seven isolates out of the 1,929 were identified as GBS $(2.4 \%$ of all isolates and $3.7 \%$ of all positive cultures). The profile of GBS isolation is summarized in Table 2. As shown in the table, twenty-eight strains were isolated from outpatients and the remaining nineteen were from inpatients, the results indicating a higher isolation ratio from oupatients $(4.5 \%$ positivity) than from inpatients $(1.4 \%$ positivity). Also, forty-four strains were recovered from female urine but only three isolates were from male urine. However, the majority of positive urine for GBS were mixtures with enterobacteriaceae or group D streptococci, that is, thirty-eight positive GBS urine out of forty-seven were mixtures with other organisms. Out of these mixed cultures, GBS were isolated as major organisms in twenty-one positive samples, and as minor organisms in ten urine samples in semi-quantitative cultures.

\section{GBS isolation from vaginal swabs and newborns}

A total of 335 cervical swabs taken from non-pregnant women were examined, and out of these, sixteen revealed GBS positive (isolation ratio $4.8 \%$ ). On the 
TABLE 2. Isolation of group $B$ streptococci from urine, vaginal swabs and newborn babies

\begin{tabular}{|c|c|c|c|}
\hline \multicolumn{4}{|l|}{ Urine } \\
\hline Quantitative culture positive* & $1,287 / 3,780$ & specimens & $(34 \%)$ \\
\hline Group B Streptococcus positive & $47 / 1,929$ & & $(2.4 \%)$ \\
\hline from outpatients & $28 / 618$ & & $(4.5 \%)$ \\
\hline from inpatients & $19 / 1,311$ & & $(1.4 \%)$ \\
\hline from female & $44 / 47$ & isolates & $(94 \%)$ \\
\hline from male & $3 /$ & & $(6 \%)$ \\
\hline isolated as major population & 21/ & mixed cultures & \\
\hline as minor population & $10 /$ & & \\
\hline equally as the other bacteria & $7 /$ & & \\
\hline \multicolumn{4}{|l|}{ Vaginal swabs } \\
\hline Isolation from pregnant women & $11 /$ & swabs & $(2.9 \%)$ \\
\hline Isolation from non-pregnant women & $16 / 335$ & & $(4.8 \%)$ \\
\hline \multicolumn{4}{|l|}{ Newborn babies } \\
\hline Isolation from throat, ear and/or nasal swabs & 106 & & $(1.9 \%)$ \\
\hline
\end{tabular}

* MS-2 urine screening was employed and positive culture means $10^{5}$ colony forming units per $\mathrm{ml}$ or more bacterial counts.

other hand, eleven isolates identified as GBS were recovered from 377 cervical swabs taken from pregnant women in their 37-40 week of gestation (isolation ratio $2.9 \%$ ). The isolation ratio of non-pregnant women was somewhat higher than from pregnant women, but not significantly. Only two cases of newborns delivered from GBS positive mothers were positive for GBS; one was positive in ear, throat and nasal swabs and the other only positive in pharyngeal swabs. But none manifested any clinical signs or symptoms of GBS infection (see Table 2).

\section{Antibiotic susceptibility}

A total of 40 strains of GBS, comprising 20 strains from urine, 18 from cervical swabs and 2 from throat swabs from newborns, were tested against eight

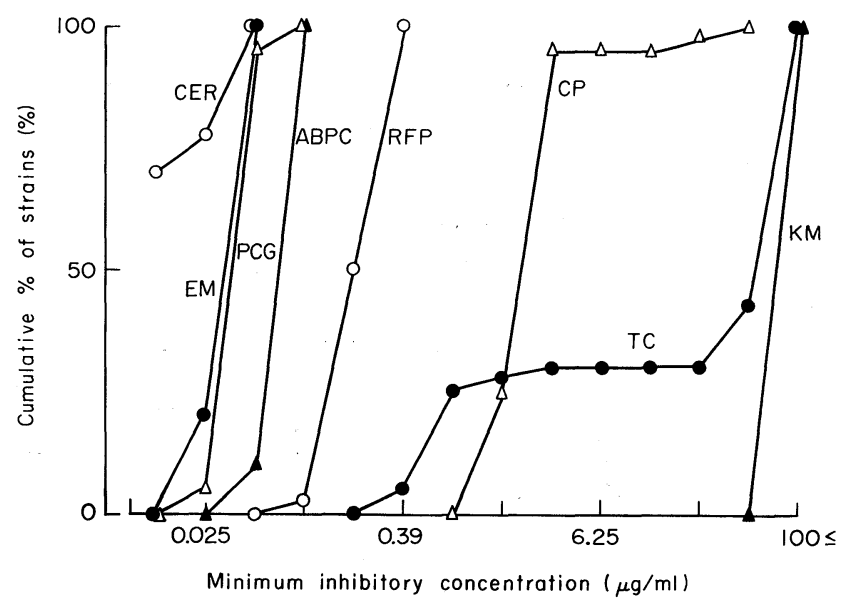

Fig. 1. Susceptibility distributions of group B streptococci against eight different antibiotics. 
antibiotics (kanamycin, KM; tetracyclin, TC; chloramphenicol, CP; rifampicin, RFP; ampicillin, ABPC; penicillin G, PCG; erythromycin, EM; cephaloridin, CER). All the isolates tested were highly sensitive against PCG, CER, and EM, and showed less than $0.05 \mu \mathrm{g} / \mathrm{ml}$ of MIC. The MICs of all the GBS ranged from 0.05 to $0.1 \mu \mathrm{g} /$ $\mathrm{ml}$ to $\mathrm{ABPC}$, and from 0.1 to $0.39 \mu \mathrm{g} / \mathrm{ml}$ to $\mathrm{RFP}$, but all the isolates were highly resistant to KM. We found two different groups of MIC values against TC and $\mathrm{CP}$, that is, almost half of the isolates were resistant to $\mathrm{TC}$ with over $50 \mu \mathrm{g} / \mathrm{ml}$ of MIC and two strains had over $25 \mu \mathrm{g} / \mathrm{ml}$ of MIC against CP. The latter two strains were also highly resistant to TC (see Fig. 1).

\section{Serotyping of GBS and their distribution}

Sixty-eight strains out of seventy-two isolates tested could be serotyped according to the method of Lancefield (1934) modified to include R, X and Ic antigens (Pattison et al. 1955a, b; Wilkinson and Eagon 1971). The serotypes of the most frequent isolates from urine were Ia $(37 \%)$ and Ic $(21 \%)$; whereas serotypes of III and III-R were found in $46 \%$ of the isolates of the vaginal swabs and serotype Ic in $29 \%$. It became apparent that serotypes of III and III-R were predominant in vaginal flora instead of Ia in urine, and that serotype Ic was highly prevalent in both urine and vaginal swabs (see Table 3). On the other hand, the results of serotyping by agglutination using heat-labile and heat-stable antigens are listed in Table 4 with the results of Lancefield's classification. As for the heatstable polysaccharide antigens, Ia, Ib, II and III, almost all the isolates tested were shown to possess antigens identical to Lancefield's classification, but some strains were shown to possess heat-labile protein antigens as well as heat-stable antigens. Out of nineteen isolates of serotype Ia, fourteen strains possessed only heat-stable Ia antigen, but the remaining three and two strains were reactive to heat-labile W and S antigen. Also, out of fourteen isolates of serotype Ic, nine strains possessed heat-labile antigens, Q, S, or W, besides Ia antigen. However, we could not find a definite difference between serotypes III and III-R classification

TABLE 3. Distribution of Lancefield's serotypes of group B streptococcus isolated from urine, vaginal swabs and newborns

\begin{tabular}{|c|c|c|c|c|c|c|c|c|c|}
\hline \multirow{2}{*}{ Origin of specimens } & \multicolumn{9}{|c|}{ Serotype } \\
\hline & Ia & $\mathrm{Ib}$ & Ic & II & III & $\mathrm{III} / \mathrm{R}$ & $\mathbf{R}$ & $\mathrm{X}$ & N.T.* \\
\hline Urine $(N=46)$ & 17 & 4 & 10 & 1 & 3 & 4 & 4 & & 3 \\
\hline \multicolumn{10}{|l|}{ Vaginal swabs } \\
\hline from pregnant $(N=10)$ & 1 & & 3 & & 2 & 3 & & & 1 \\
\hline from non-pregnant $(N=14)$ & 2 & 1 & 4 & 1 & 3 & 3 & & & \\
\hline Subtotal & 3 & 1 & 7 & 1 & 5 & 6 & & & 1 \\
\hline Newborns $(N=2)$ & & & 1 & & 1 & & & & \\
\hline Total & 20 & 5 & 18 & 2 & 9 & 10 & 4 & 0 & 4 \\
\hline
\end{tabular}

* N.T., non-typable. 
TABLE 4. An alternative serotyping of group $B$ streptococcus by heat-stable and heat-labile antigens

\begin{tabular}{ccc}
\hline $\begin{array}{c}\text { Lancefield's } \\
\text { serotype }\end{array}$ & $\begin{array}{c}\text { The alternative } \\
\text { serotype }\end{array}$ & $\begin{array}{c}\text { Number of isolates } \\
\text { identified }\end{array}$ \\
\hline Ia & Ia & 14 \\
& Ia/W & 3 \\
Ib & Ia/S & 2 \\
& Ib & 1 \\
Ic & Ib/S & 3 \\
& Ia & 1 \\
& Ia/Q & 5 \\
II & Ia/S & 5 \\
III & Ia/W & 3 \\
& II & 1 \\
& III & 1 \\
III/R & $\mathrm{R}$ & 5 \\
& III/R & 2 \\
& III/X & 1 \\
R & III/R & 1 \\
& III & 4 \\
& $\mathrm{R}$ & 3 \\
N.T.* & $\mathrm{R}$ & 1 \\
& $\mathrm{Q}$ & 2 \\
& $\mathrm{R}+\mathrm{Q}$ & 1 \\
& $\mathrm{Q}$ & 1 \\
& $\mathrm{~S}+\mathrm{W}$ & 1 \\
& $\mathrm{~N} . \mathrm{T} . *$ & 1 \\
& & 2 \\
\hline
\end{tabular}

* N.T., non-typable.

when we employed the new nomenclature system. Out of four strains which could not be serotyped by Lancefield's classification, two strains possessed heatlabile $\mathrm{Q}$ and $\mathrm{S}+\mathrm{W}$ antigens and two strains remained untypable.

\section{Discussion}

Our study described in this communication was first initiated to estimate the ratio of group B streptococci among Japanese women. At our hospital, GBS were recovered from vaginal swabs taken from pregnant women with the isolation ratio of $2.9 \%$. The prevalence of GBS obtained was thought to be less than that in other Western countries ranging from 4 to $20 \%$ previously reported (Franciosi et al. 1973; Anthony et al. 1981), but almost the same as the ratio among Mexican pregnant women (Collado et al. 1981). Our results also strongly suggest that the prevalence of GBS markedly differs reflecting differences in human genetic constitution. That is to say, GBS infection and carriage ratio seems to be far less frequent among Mongolians compared with Caucasians and Negroes. Although disease due to GBS in neonates has not emerged in Japan, our study was conducted for vaginal swabs only, and it is becoming apparent that the origin of GBS in birth 
canal is closely related with carriage in the intestine (Badri et al. 1977; Hammerschlag et al. 1977; Maurer et al. 1979; Dillon et al. 1982). It will be necessary to check therefore how isolation ratios from anorectal swabs are compared with the ratios observed in Western countries. A GBS isolation ratio of 3.7\% was recovered from urine positive for bacterial counts. Although there have been several reports concerning group B streptococcal bacteriuria, their clinical significance is still unclear (Mhalu 1977; Bollgren et al. 1978; Wood and Dillon 1981). Although GBS was sometimes the major bacteria population in urine, it was almost always isolated from mixture of bacteria including Escherichia coli and/or enterococci. The clinical features were characteristic; almost all the GBS were isolated from female urine and successful isolations were mainly observed in outpatients urine. These findings suggest the ano-genito-urethral transmission of GBS on female perineal skin and possible sexual transmission from the female carrier to her male sexual partner (Franciosi et al. 1973; Gardner et al. 1979).

Serotyping information on GBS isolates is now widely used in clinical laboratories, and provides a reasonable marker to confirm the association between vaginal carriage and neonatal GBS infection. Lancefield (1938) originally established four different polysaccharide antigens, Ia, Ib, II and III in acid-heat extracts, and then, Pattison et al. (1955a, b) added the protein antigens $\mathrm{R}$ and $\mathrm{X}$ to Lancefield's scheme. Also, protein antigen Ic was most recently established by Wilkinson and Eagon (1971). However, the procedure described by Lancefield (1938) is complex and time-consuming, and also, unreasonable cross reactions and weakened reactivity during passages have been pointed out. These problems prompted us therefore to develop an alternative approach to serotyping. The method described in this communication is based on independently determining the kind of heat-labile protein antigen and heat-stable polysaccharide antigen present in the GBS. In our laboratory, we identified four different heat-stable polysaccharide antigens (Ia, Ib, II and III) and five heat-labile protein antigens $(\mathrm{S}, \mathrm{X}, \mathrm{R}, \mathrm{Q}$ and $\mathrm{W})$ in various protein/polysaccharide combination forms (i.e., $\mathrm{Ia} / \mathrm{W}, \mathrm{Ib} / \mathrm{S}$, etc). In other words, within each of Lancefield's major serotype groups, we found several distinct varieties, some of which possessed only antigens corresponding to Lancefield's serotyping and others possessed both protein and polysaccharide antigens. Although this alternative system is still tentative, we do not foresee any problems in incorporating our two unidentified isolates or any new antigens, once they are serotyped. Thus the alternative nomenclature system provides an easy and detailed procedure to identify the serotypes of GBS. This effort will contribute to the serological analysis of GBS and will give us further information on the association of GBS in vaginal and/or rectal flora, and perinatal infections. 


\section{Acknowledgments}

We would like to thank Dr. H. Yokoo for donating antisera and his thoughtful suggestions, and Dr. Richard R. Facklam and Dr. J. Rotta for donating antisera to reference strains of group B streptococci. This work was supported in part by a grant from the Sendai Microbiology Institute.

\section{References}

1) Anthony, B.F., Okada, D.M. \& Hobel, C.J. (1978) Epidemiology of group B Streptococcus: longitudinal observations during pregnancy. J. Inf. Dis., 137, 524-530.

2) Anthony, B.F., Eisenstadt, R., Carter, J., Kim, K.S. \& Hobel, C.J. (1981) Genital and intestinal carriage of group B streptococci during pregnancy. J. Inf. Dis., 143, 761-766.

3) Badri, M.S., Zawaneh, S., Cruz, A.C., Mantilla, G., Baer, H., Spellacy, W.N. \& Ayoub, E.M. (1977) Rectal colonization with group B Streptococcus: relation to vaginal colonization of pregnant women. J. Inf. Dis., 135, 308-312.

4) Bollgren, I., Vaclavinokova, V., Hurvell, B. \& Bergqvist, G. (1978) Periurethral aerobic microflora of pregnant and non-pregnant women. Brit. med. J., 1, 1314-1317.

5) Butter, M.N. \& de Moor, C.E. (1967) Streptococcus agalactiae as a cause of meningitis in the newborn, and of bacteraemia in adults: differentiation of human and animal varieties. Antonie Van Leeuwenhoek., 33, 439-450.

6) Centers for Disease Control (1977a) Isolation and identification of Streptococci: Part III. Presumptive identification of Streptococci by nonserological methods. U.S. Department of Health and Human Services.

7) Centers for Disease Control (1977b) Isolation and identification of Streptococci: Part II. Extraction and serological identification. U.S. Department of Health and Human Services.

8) Collado, M., Kretschmer, R.R., Becker, I., Guzmán, A., Gallardo, L. \& Lepe, C.M. (1981) Colonization of Mexican pregnant women with group B streptococcus. J. Inf. Dis., 143, 134.

9) Dillon, H.C., Jr., Gray, E., Pass, M.A. \& Gray, B.M. (1982) Anorectal and viginal carriage of group B streptococci during pregnancy. J. Inf. Dis., 145, 794-799.

10) Eickhoff, T.C., Klein, J.O., Daly, A.D., Ingall, D. \& Finland, M. (1964) Neonatal sepsis and other infections due to group B beta-haemolytic streptococcci. New Engl. J. Med., 271, 1221-1228.

11) Franciosi, R.A., Knostman, J.D. \& Zimmerman, R.A. (1973) Group B streptococcal neonatal and infant infections. J. Pediat., 82, 707-717.

12) Gardner, S.E., Yow, M.D., Leeds, L.J., Thompson, P.K., Mason, E.O., Jr. \& Clark, D.J. (1979) Failure of penicillin to eradicate group B streptococcal colonization in the pregnant woman: a couple study. Amer. J. Obstet. Gynecol., 135, 1062-1065.

13) Hall, R.T., Barnes, W., Krishnan, L., Harris, D.J., Rhodes, P.G., Fayez, J. \& Miller, G.L. (1976) Antibiotic treatment of parturient women colonized with group B streptococci. Amer. J. Obstet. Gynecol., 124, 630-634.

14) Hammerschlag, M.R., Baker, C.J., Alpert, S., Kasper, D.L., Rosner, I., Thurston, P., Webb, B.J. \& McCormack, W.M. (1977) Colonization with group B streptococci in girls under 16 years of age. Pediatrics, 60, 473-476.

15) Japan Society of Chemotherapy (1981) The standard method for minimum inhibitory concentration (MIC) assay remodified. Chemotherapy, 29, 76-79. (in Japanese)

16) Lancefield, R.C. (1934) A serological differentiation of specific type of bovine haemolytic streptococci (group B). J. exp. Med., 59, 441-458.

17) Lancefield, R.C. (1938) Two serological types of group B haemolytic streptococci with related, but not identical, type-specific substances. J. exp. Med., 67, 25-39.

18) Maurer, M., Thirumoorthi, M.C. \& Dajani, A.S. (1979) Group B streptococcal colonization in prepubertal children. Pediatrics, 64, 65-67.

19) McCracken, G.H., Jr. \& Shinefield, H.R. (1966) Changes in the pattern of neonatal 
septicemia and meningitis. Amer. J. Dis. Child., 112, 33-39

20) Mhalu, F.S. (1977) Streptococcus agalactiae in urinary tract infections. Postgrad. med. $J ., 53,216-218$.

21) Murai, T., Inazumi, Y., Oguro, H. \& Ishiguro, K. (1980) Serotype of group B streptococci isolated in Japan in recent years. Jap. J. Inf. Dis., 54, 22-28. (in Japanese with English abstract)

22) Ohashi, H. (1981) Clinical and bacteriological study on microbial flora in the vagina: (2) A study on isolation rates of group B streptococci in the vagina of pregnant and non-pregnant women. Jap. J. Inf. Dis., 55, 109-115. (in Japanese with English abstract)

23) Pattison, I.H., Matthews, P.R., Jr. \& Howell, D.G. (1955a) The type classification of group B streptococci with special reference to bovine strains apparently lacking in type polysaccharide. J. Path. Bact., 69, 51-60.

24) Pattison, I.H., Matthews, P.R., Jr. \& Maxted, W.R. (1955b) Type classification by Lancefield's precipitin method of human and bovine group B streptococci isolated in Britain. J. Path. Bact., 69, 43-50.

25) Wilkinson, H.W. \& Eagon, R.G. (1971) Type-specific antigens of group B type Ic streptococci. Infect. Immun., 4, 596-604.

26) Wood, E.G. \& Dillon, H.C., Jr. (1981) A prospective study of group B streptococcal bacteriuria in pregnancy. Amer. J. Obstet. Gynecol., 140, 515-520. 